\title{
TeamMATE: Computer Game Environment for Collaborative and Social Interaction
}

\author{
Daniel I. Thomas \\ Technology One Ltd. \\ 67 High St. Toowong 4066 QLD, Australia \\ Daniel_Thomas@technologyonecorp.com
}

\author{
Ljubo Vlacic \\ Intelligent Control Systems Laboratory \\ Griffith University \\ Nathan, 4111 QLD, Australia. \\ L.Vlacic@griffith.edu.au
}

\begin{abstract}
In order to investigate the interaction and collaboration of buman and artificial beings as fully equal partner agents, we have developed an interactive computer game called TeamMATE ${ }^{*}$ and tested its operation under a boardroom-like play scenario. We outline the key features and desirable properties that are necessary in an electronic boardroom environment and introduce a Physical Layer concept that allows us to define the electronic boardroom as well as the required sensors, effectors and communication protocols in an extensible manner. Once the basics of the TeamMATE system have been described, we demonstrate a simple brainstorming process defined within a physical layer. Within this electronic boardroom, human and artificial beings, as fully equal partner agents, interact collaboratively to fulfill the various roles and responsibilities related to the process under observation. Finally, we conclude with our observations of TeamMATE in action, and how this will contribute to further work with collaborative fully equal partner agents.
\end{abstract}

Index Terms - Autonomous agents, Collaborative agents, Electronic Boardroom, Computer games

\section{INTRODUCTION}

Computer game development offers a compelling platform for many fields of academic research. As each new game produced by the industry pushes the boundaries of technical possibility, it should come as no surprise that academia and the game industry have frequently crosspollinated each others' efforts.

In this paper, we describe the architecture of a computer game environment we have developed called TeamMATE. This environment facilitates the investigation of biological and artificial cooperative autonomous agents as fully equal parther agents. Given our interest in fully equal parther agents, we have divided our research questions into three parts:

- Can human and artificial beings, being heterogeneous agents, interact cooperatively as fully equal partners in the context of computer games, where each entity is fully replaceable or substitutable with the other?

- How can cooperation be obtained in heterogeneous agent situations, such as a boardroom, being designed to facilitate cooperation between biological and artificial agents?

* Copyright ICSL
- Can a boardroom-like game scenario be appropriate for social and educational computer games?

In this paper, we will address the first research question and the TeamMATE system we have developed in response to it.

The paper is organized as follows. Part Two describes the fully equal partner agent concept. Part Three outlines the features of a computer game environment to facilitate the interaction between fully equal partners. Part Four defines the physical layer concept that allows TeamMATE to describe different boardrooms. Part Five demonstrates a brainstorming boardroom defined within TeamMATE using a physical layer definition. Part Six concludes with some of our thoughts on further work and application of this concept.

\section{Agents AS FUlly EQUAL PARTNERS}

While there are many different definitions of what can be defined as an agent, we prefer to use the simple notion of agency as described by Jennings and Wooldridge [1]. In our work, we also divide agents into Biological (Humans) and Artificial (Software, Robots etc) agents.

We see a fully equal partner concept where biological and artificial agents collaborate to achieve game goals as complementary to other uses of agents as opponents [2] or as interactive story characters [3] (also known as NonPlayer Characters or NPCs). Non-player characters are typically able to work with (or provide simple assistance to) the human players, but do not participate as intelligent collaborative entities equal in ability to a biological agent.

We propose using cooperative autonomous agents as fully equal partners in a computer game scenario. This means that any agent (biological or artificial) is completely replaceable by either a biological (human) agent or an artificial agent (Figure 1).

A fully equal partner agent:

- Does not behave as though humans playing the game are the centre of attention;

- Can work cooperatively with other fully equal partner agents (including humans) and is not always adversarial;

- "play" the game as a human would; and

- Does not work to a defined script or take direction from an agent "director" [3, 4]. 
Put in more concisely, a fully equal partner agent (biological or artificial) performs tasks cooperatively with other agents and is fully replaceable or interchangeable with another fully equal partner agent. This also means that an agent does not know whether his/her game partner is a human or artificial being.

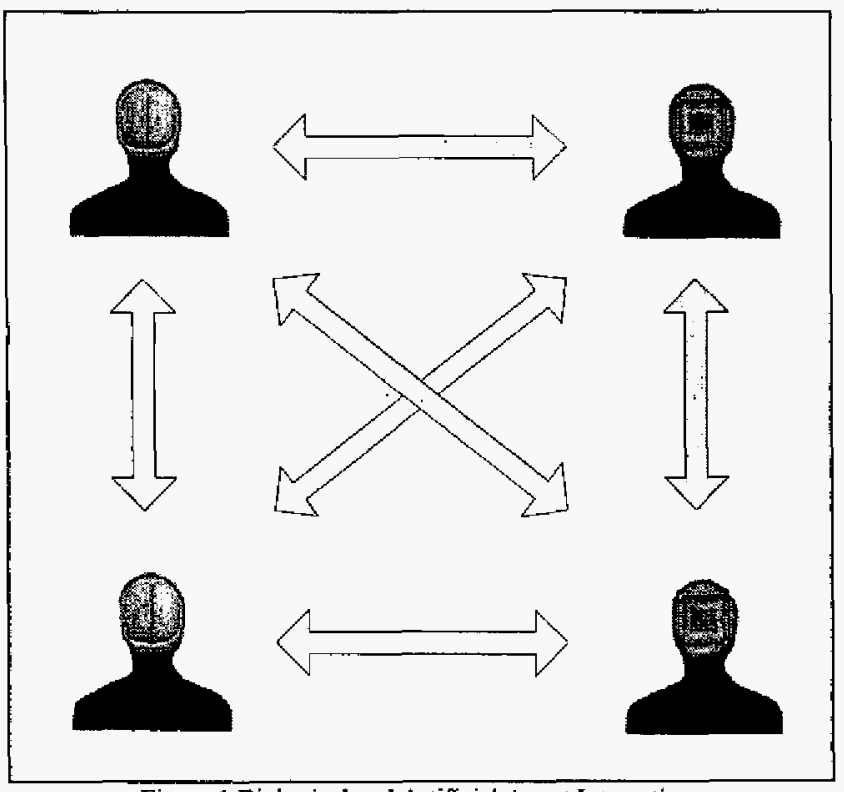

Figure 1 Biological and Artificial Agent Interaction

\section{ENVIRONMENT REQUIREMENTS}

In order to describe the TeamMATE concept, it is necessary to define what we consider the key requirements of a computer game capable of supporting fully equal partner agents.

\section{A. Key Features}

There have been a number of efforts to classify and evaluate simulation environments for agent research $[5,6]$. In our earlier work [7] we set out to evaluate a number of computer games in terms of their suitability for cooperative autonomous agent research in the context of computer games and social interactions. This work resulted in the definition of three groups of key features that identified the essence of a desirable simulation environment. We have used these key features to guide the architectural requirements of the TeamMATE system.

The system that we have developed is designed to facilitate the interaction between human and artificial beings as Fully Equal Parthers. TeamMATE is a software system based around the concept of facilitating interaction by situating biological and artificial agents in a common environment. In our case, the environmental concept that we have selected is called the Electronic Boardroom. From this concept we have focused our interest on mental (and cognitive) games. To accomplish this task, our system can be divided into three distinct parts (figure 2):

- A mechanism that allows biological agents to situate themselves in the electronic boardroom;
- A mechanism that allows artificial agents to situate themselves in the electronic boardroom and;

- The Electronic Boardroom environment itself.

From these three core components of the TeamMATE system it was possible to determine what was required in terms of the desirable features of a computer game utilizing the fully equal partner (and cooperative) agents concept.

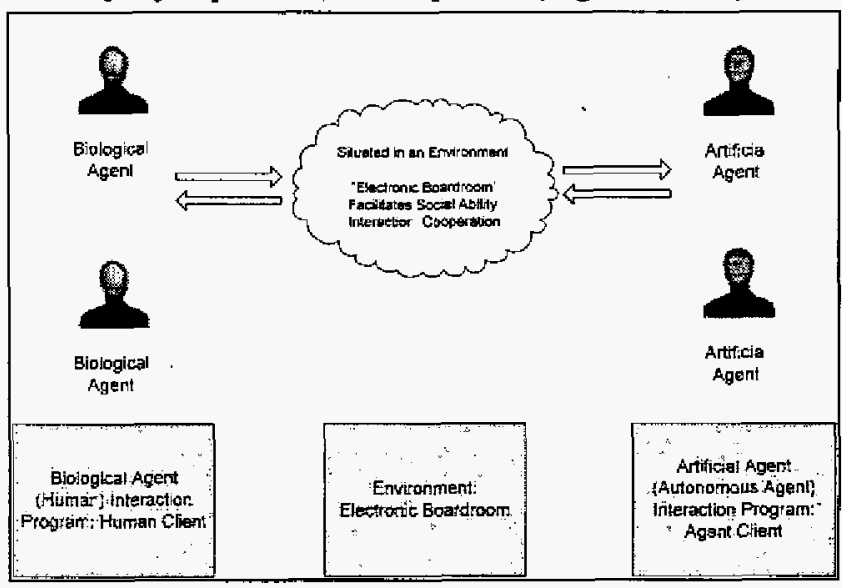

Figure 2 Decomposing the required conceptual elements into highlevel software systems.

The core components of TeamMATE were designed around the key requirements determined by our earlier work. There are three groups of requirements divided as follows:

- Features of the simulation environment that support cooperative autonomous agents

- Features required by cooperative agents situated within this environment

- The practical considerations related to the implementation of such a simulation environment.

\section{B. Key Requirements of the Boardroom}

When defining the key features of a simulation environment that supports fully equal partner agents, the following key features were found to be the most desirable $[8,9]$

- Exogenous Events

- Causal Structure

- Notion of Time

- Support for Experimentation [10]

- A clean well-defined interface between the environment and agents that are situated within it.

In our case, the boardroom must support the ability to place unplanned/unexpected events within the given scenario, such as stock market fluctuations, shipping delays for goods, new competitors or any of a myriad of events that our electronic boardroom members must work through. This feature is to utilize the given scenario's physical layer elements to create these unplanned events. Behavior should occur in two ways: controlled and uncontrolled.

Controlled events are events that can be described as unexpected by the agents, but are initiated during an 
experiment by researchers controlling the boardroom scenario. Uncontrolled events are to occur without the intervention of a researcher or other external entity.

The boardroom scenarios must define rules at the physical layer level about the scenario "world". These relationships define how the elements within the boardroom can be manipulated by an agent, and what the effects are upon the surrounding elements/agents.

All elements of the system are to support experimental use; mechanisms including recording and playback of scenario actions are essential to compare and contrast our work with that of others.

While we maintain that a real-time system is most desirable, it does not always fit a given scenario (for example, a scenario that would in a "real time" take one year). For this reason, we have relaxed this concept of time to encapsulate discrete time steps and turn-based temporal concepts. The definition of time is specified as part of the physical layer definition of the boardroom.

\section{Key Requirements of the Agent}

During the course of this research, we adopted the definition of an agent from that presented by Jennings and Wooldridge $[1,11]$. An agent is:

- Autonomous

- Situated in an environment, aware of its surroundings

- Capable of some form of Social Ability

- Reactive

- Proactive

The TeamMATE system is developed with complete autonomy of the agents in mind. While the agents are situated in their environment and are able to utilize various sensors and effectors, they are completely autonomous entities. We deliberately separate agents from the environment through the use of a network communications layer. This also has the advantage of allowing us to utilize distributed hardware such as PCs connected to a Local Area Network, Wide Area Network or the Internet. Of course, there is nothing stopping a number of entities operating on the same workstation. For this reason, we required a network communication layer

In order to interact and cooperatively achieve goals, the system required a Social Ability [1], or the ability to communicate in meaningful ways with other agents in their environment. In order to achieve this and effectively support experimentation within our environment we divided this necessary feature into distinct layers. Each layer is substitutable with another equivalent technology, allowing us to compare and contrast:

- Basic Communication Layer (in our case, a language used to transmit and receive data from the boardroom)

- A Physical Layer which contains the necessary building blocks to describe, sense and effect environmental objects in a given boardroom environment

- Finally, a Cognitive Layer which contains language to describe abstract concepts (a future objective).

Agents situated in the boardroom should react in a timely manner. For this reason, agents must respond in a timely manner to information passed to their sensors. Efficient evaluation and response is of major consideration, as well as the efficiency of the mechanism that provides the sensors with information. In our case, this requires the effective interpretation of sensor data as well as contextual circumstance.

In order for agents to exhibit pro-active behavior in pursuing their objectives, it is necessary to provide a mechanism to supply these objectives in the first place. Objectives shall be divided into two categories:

- those defined as part of the physical layer and;

- those defined as part of the cognitive layer.

At this point in time, physical layer objectives are supported, with cognitive layer properties specified for the next phase of our work going forward.

\section{Key Practical Requirements}

From a practical point of view, the following features affect the benefits of a simulation environment as much as features from the other groups [5]:

- Documentation

- World Complexity

- Support (Technical)

- Platform Availability

- Method of Distribution

Documentation is being made available for TeamMATE usage. This should also allow other researchers to test our work or utilize the system in their own work.

Environmental complexity is supported by the concept of the physical layer. Scenarios may be as simple or complex as desired by the scenario being undertaken.

For development of TeamMATE itself, there is a high abundance of relevant and useful documentation and support for using .NET and DirectX technologies.

Our choice of development platform was subjective based on our experience with these technologies. In the context of our objectives, we believe that utilizing these tools would allow us to complete our goals in the most effective manner.

As part of the development process, we needed to select technologies that would be most effective in bringing the TeamMATE system to life:

- To form the backbone of our communications layer, we selected DirectPlay, a component of Micorsoft Direct X; a suite of tools and libraries used in the development of computer games.

- By using Microsoft's . Net technologies, we were also able to utilize the integrated development environment Visual Studio. This tight integration of .Net and 
development environment allowed us to rapidly establish the framework of the TeamMATE system.

\section{THE PHYSICAL LAYER}

The physical layer of the TeamMATE system is one of three layers that the electronic boardroom uses (Figure 3).

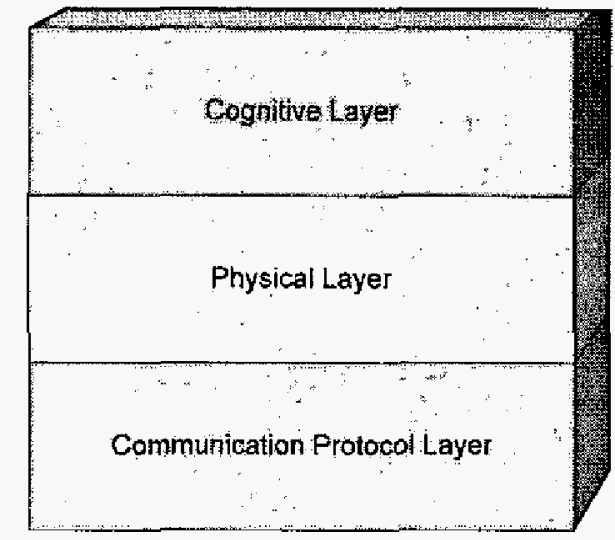

Figure 3 Communication, Physical and Cognitive layers of the electronic boardroom

The communication protocol layer is effectively a low level transport layer used to pass information from one place to another (for example: DirectPlay, TCP/TP, radio signal etc). Simplistically, the physical layer and the cognitive layer can be thought of as the "language" and "intent/meaning" layers respectively. For the purposes of this paper, we will focus on the physical layer.

Before we are able to work with more abstract mental layers (which we call Cognitive Layers) in the electronic boardroom, it was necessary to define a layer that was:

- Able to define the physical objects of the virtual world (the electronic boardroom);

- Provide a common communication protocol for members of the boardroom,

- Defines the possible actions that may be performed within the electronic boardroom;

Fully equal partner agents must be able to work with the appropriate rules/constraints of the specific play scenario being undertaken. Depending on the play scenario being undertaken, the physical layer mechanisms are "plugged" into the TeamMATE system. These physical layers:

- Define available sensors

- Define available effectors

- Boardroom world environment physical rules.

Physical rules for a given boardroom play scenario consist of information about objects in the environment and how they may be used. Take as an example, a simple play scenario could contain these physical layer rules:

TABLE 1

\begin{tabular}{|l|l|}
\multicolumn{1}{|c|}{ EXAMject } & \multicolumn{1}{c|}{ Available Actions } \\
\hline Chair & Sit, Stand, Move \\
\hline Table & Place Item, Pick Item \\
\hline Telephone & Call, Hang Up, Speakerphone, Listen \\
\hline Stock & Buy, Sell, Report \\
\hline Talk & Whisper, Tell All, Listen \\
\hline
\end{tabular}

Currently, physical layers are available for selection when loaded. It is our intention to automate this process and deliver any required physical layer interface elements via boardroom communication when joining a play scenario.

While the electronic boardroom and the fully equal parther agents that are involved in a given play scenario share a common physical layer, it is not necessarily true that the manifestation of the physical layer will be the same.

\section{E. An Electronic Boardroom Meeting}

While our boardroom-like computer game concept has been designed to operate without the assistance of an agent "director", we do have one key authority role: The Chairperson.

The chairperson typically is responsible for the organization, initiation and conclusion of a boardroom-like meeting. Depending on how an electronic boardroom work environment has been defined, other roles can be defined as part of the physical layer definition.

The boardroom-like discussion and decision-making process consists of the following elements:

- Initiation

- Conversation

- Actions

- Conclusion

At a point determined by the chairperson (typically when all participants are present, or the scheduled meeting time has been reached). The chairperson will declare the meeting "started". It is at this point that the electronic boardroom meeting can commence. Initiation of the electronic boardroom meeting is actually a special action (we describe actions in more detail later).

All communication and collaborative behavior within the electronic boardroom take place in the form of "Conversations". The first part of any conversation is the Question. Questions in the electronic boardroom are any statements that result in an outcome (statements or orders are also considered "questions"). When an agent proposes a question, there are three possible outcomes:

- A response (which may be itself another question)

- An action

- No Response

Actions are special responses to questions that result in a transition of some item or process from one state to another. For example, if a participant asked the question "I require a technician for Project $X^{\prime \prime}$, a possible resulting outcome may be that another participant in the boardroom may perform an action that results in the commencement of a recruitment process to hire a skilled technician for Project $\mathrm{X}$.

At either a specified time, or when the objectives of the meeting have been completed successfully, the chairperson is able to enact a special action that concludes the electronic boardroom meeting. 


\section{Play Scenario: A Brainstormtng Session}

In demonstrating our work, a brainstorming session is chosen as the most appropriate scenario. This scenario demonstrates a simple electronic boardroom used to obtain a collaborative outcome.

In this play scenario, we have five agents: Daniel, Lucy, George, Frank and Paul. The members of the boardroom face a tough decision, that of choosing a destination for a holiday. Each of the board members has particular attributes that they desire in a holiday. George, as chairperson has organised a brainstorming meeting to choose a holiday destination that all board members can agree upon.

\section{F. A Collaborative Process}

The intention of a collaborative process is to demonstrate that a physical layer is capable of facilitating interaction and collaboration through the defined communication processes and action mechanisms.

In this simple example of a physical layer, the stages of the brainstorming process are as follows:

- The chairperson initiates the meeting

- The chairperson describes the goal of the meeting (choosing a holiday).

- The chairperson then presents a number of holiday options to the other members.

- Each member in turn performs a rate action (Like, Dislike or Neutral) and may give a reason for their action.

- Other members may agree/disagree with the member.

- The chairperson may then use these reasons to present another holiday destination.

- This process is repeated until a set limit of holidays is presented (for example 5 destinations)

- The chairperson then informs the group which holiday destination will be selected based on a "best fit" of board member likes/dislikes.

- The chairperson concludes the meeting.

\section{G. TeamMATE in Action}

In this simple example, there are a small number of actions that are defined in the physical layer that are utilized by the agents:

The physical layer defines two roles for this exercise: The chairperson and the Board member:

TABLE 2

BRAINSTORMING BOARDROOM ROLES AND DEFINED ACTIONS

\begin{tabular}{|l|l|}
\hline \multicolumn{1}{|c|}{ Role } & \multicolumn{1}{|c|}{ Actions } \\
\hline Chairperson & Initiate Boardroom \\
& $\begin{array}{l}\text { Present Holiday Option } \\
\text { Close Boardroom }\end{array}$ \\
\hline Board member & Like Holiday \\
& Dislike Holiday \\
& Neutral \\
\hline
\end{tabular}

For this exercise, we had very limited free-form conversation support (for the collection of like/dislike reasons).

In Figure 4, the TeamMATE electronic boardroom can be seen in action. In this screenshot, we see the boardroom from the perspective of the biological agent Daniel. The means by which Daniel interacts with the boardroom is the interaction toolbar at the bottom of the screen. The defined actions that are available to Daniel are the actions defined by the board member role. Likewise, Lucy, Paul and Frank (which are either human or artificial beings) also have the actions defined by the board member role. It should be also noted that from the perspective of all board members, the chairperson (George) is positioned at the head of the table.

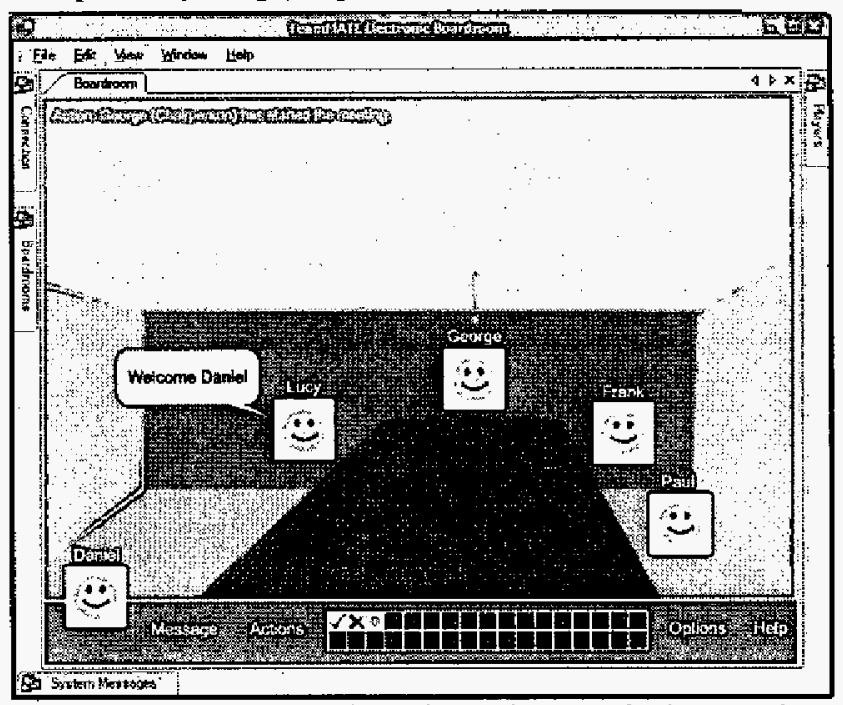

Figure 4 TeamMATE Electronic Boardroom conducting a meeting

George on the other hand, is acting in the role of the chairperson and has the actions defined by the specialized brainstorming chairperson role. These actions like those of Daniel are presented in the actions toolbar.

If George was not actually a biological agent but an artificial one, there is no user interface (as a biological agent would perceive it) as the interface with its defined sensors and effectors is defined within the artificial agent application (Figure 5). Thus, Daniel believes that all the agents of concern are human beings although either one of them or all of them can be artificial ones.

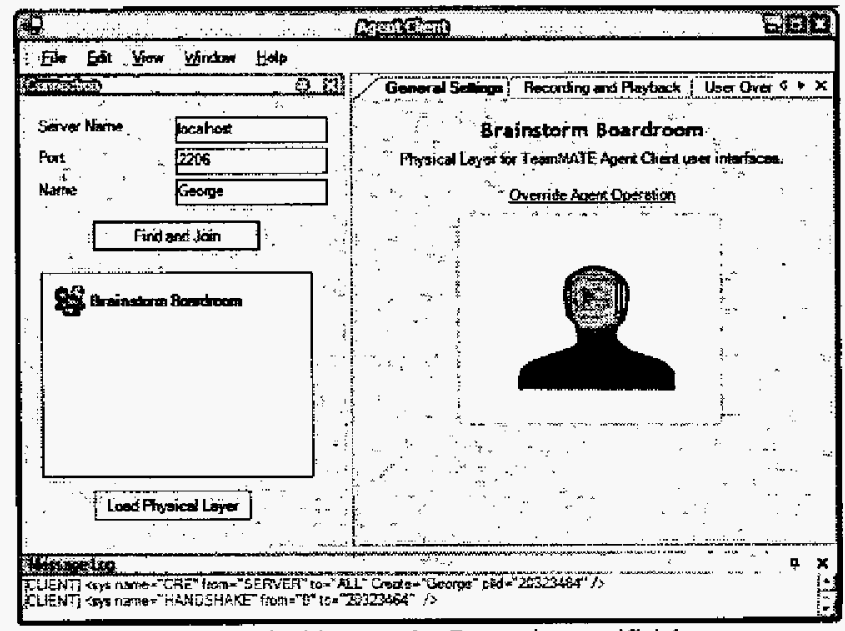

Figure 5 in this example, George is an artificial agent 


\section{H. Further Work}

As we introduce more complex cognitive layers to the system, a mental nature of the play scenario, i.e. conversational interactions and collaboration among the agents will become more important. For example: in our brainstorming holiday scenario, the negotiation would become more complex between the agents as each entity is capable of debate, compromise, renegotiation and the use of prior decisions to choose a holiday destination.

In this scenario, each biological and artificial agent is represented by an avatar in the form of a face. As part of our further work, we intend to include in the physical layer definition, a means by which each agent may visually "emote" by changing their avatar's look (for example: happy and sad emotions) that can be interpreted by other members of the boardroom (rather than the simple like/dislike actions used in our example).

Another static element in the boardroom was the room and boardroom table. In future work, the boardroom dimensions and other elements should be defined in the physical layer, providing a rich agent environment for interaction.

\section{CONCLUSion}

We have presented the TeamMATE system concept that provides both biological and artificial agents as fully equal parthers an environment for collaborative interaction.

Firstly, the key features and desirable properties that are necessary in an adequate simulation environment were outlined. We then used these requirements to define and elaborate on the TeamMATE system.

We described the Physical Layer concept, which allows us to define the electronic boardroom scenario as well as the required sensors, effectors and communication protocols. We described how this allows us to develop more sophisticated boardrooms as we progress in our work.

By putting these concepts together, we demonstrated a simple brainstorming game play session used by biological and artificial agents. The physical layer consists of basic physical elements, sensors and effectors that the board members utilise to achieve the goals of the brainstorming session.

We also find potential for this system in developing online entertainment, in order to enhance the features and roles of non-human entities in Massively Multiplayer Online Games.

The next phase of our work will introduce the complex concept of cognitive layers to TeamMATE, as well as accentuating the collaborative nature of an electronic boardroom to study fully equal partner agents in training and simulation games.

\section{References}

[1] Jennings, N. R. and Wooldridge, M. Intelligent Agents: Theory and Practice Krowledge Engineering Review; 1995, 10

[2] Laird, John E Using a Computer Game to Develop Advanced AI IEEE Computer, 2001, 34, 70-75
[3] Magerko, Brian and Laird, John E. and Assanie, Mazin and Kerfoot, Alex and Stokes, Devvan AI Characters and Directors for Interactive Computer Games Proceedings of the 2004 Innovative Applications of Artificial Intelligence Conference, 2004

[4] Riedl M, Saretto C.J. and Young R. M. Managing interaction between users and agents in a multiagent storytelling environment, in the Proceedings of the Second Intemational Conference on Autonomots Agents and Multi-Agent Systems, June, 2003

[5] Thomas, D and Vlacic, $L$ Selecting an Environment for Cooperative Autonomous Robot Research Intelligent Robots: Vision, Learning and Interaction KAIST Press, 2003 , 187-198

[6] Ejter, Thomas and Mascardi, Vjviana Comparing Environments for Developing Software Agents A/ Commonications, 2002, I5, 169-197

[7] Thomas, D and Vlacic L Balancing Fun and Facts: Developing a Computer Game for Research and Entertainment Microelectronic Research Conference (MERC'03) 2003, also ICSL Research Report No 2003/6I

[8] Cohen P. R, Hanks S., Pollack M. E., Benchmarks, Testbeds, Controlled Experimentation, and the Design of Agent Architectures, AI Magazine, 14(4), pp 17-42, 1993. Winter Issue.

[9] Horling B., Lesser V., Vincent R., Experiences in Simulating MultiAgent Systems Using TAMS, The Fourth Intemational Conference on Multiagent Systems (ICMAS 2000), Boston MA, July, 2000, AAAI. Also submitted as UMASS tech report 1999-75.

[10] Kaminka, G. A.; Veloso, M; Schaffer, S.; Sollitto, C.; Adobbati, R.; Marshal, Andrew N.; Scholer, Andrew, S.; and Tejada, S. 2002. GameBots: the ever-challenging mult-agent research test-bed, In Communications of the ACM, January issue

[11] Wooldridge M., Agent-Based Software Engineering, IEEE Transactions on Software Engineering, 144(1), pp26-37, February 1997.

[12] Lehman, J. F., Laird, J. E., \& Rosenbloom, P. S. (1996). A gentle introduction to Soar, an architecture for human cognition. In $S$. Stemberg \& D. Scarborough (Eds.), Invitation to cognitive science, vol. 4. Cambridge, MA: MTT Press. 